\title{
CAP and HCAP are different? An unresolved question
}

We read with interest the recent article by Polverino et al. ${ }^{1}$ Data of this study show a very low incidence of multidrug-resistant pathogens (MDR) in patients with community-acquired pneumonia (CAP) and healthcare-associated pneumonia (HCAP); authors conclude that microbial aetiology of HCAP does not differ from CAP, and that patients with HCAP in Spain do not need nosocomial antibiotic coverage. Surprisingly, the reported data conflict with a recent prospective study by Giannella et $a l^{2}$ conducted in 72 internal medicine wards in Spain, and enrolling 1002 patients; in this study Pseudomonas aeruginosa and methicillin-resistant Staphylococcus aureus caused $17 \%$ and $12.3 \%$ of HCAP, respectively; moreover, HCAP was an independent risk factor for pneumonia due do difficult-to-treat micro-organisms.

How can these differences be explained?

The substantial differences observed in two large multicentric populations of a same country may be indicators of two important problems: (1) since most patients 
of the study of Polverino et al were hospitalised in pneumology wards this may introduce a selection bias in term of epidemiology, diagnostic procedures and outcomes; patients hospitalised in internal medicine wards may be more frequently affected by multiple comorbidities and more likely to be repeatedly exposed to the healthcare setting; ${ }^{3}$ (2) in both studies routine sampling included blood cultures, urine antigens and sputum cultures but patients were not prospectively assigned to invasive diagnostic procedures like bronchoscopy with bronchoalveolar lavage cultures; as matter of fact, an aetiology was obtained in a minority of cases $(34.9 \%$ and $26 \%$, respectively), and a large proportion of patients with HCAP and CAP remained without diagnosis. The importance of an aggressive diagnostic approach was demonstrated by Lacroix et al, ${ }^{4}$ who, using the early mini bronchoalveolar lavage in the diagnosis of HCAP, identified a microbial aetiology in $46.3 \%$ of cases, and when the patient did not receive antibiotic therapy before the procedure pathogens were identified in $72.6 \%$ of cases $(16 \%$ of cases had a MDR aetiology). This experience suggests that an aggressive diagnostic approach may be more accurate to assess aetiology of CAP and HCAP.

Since data about microbiology of CAP and HCAP are contradictory, probably we need future interventional studies to define the role of MDR pathogens in patients with community-onset pneumonia. Until then, an individualised risk assessment for MDR organisms appears reasonable in patients fulfilling HCAP definition, ${ }^{5}$ to determine which patients presenting with pneumonia may require broad-spectrum antibiotic coverage.

Marco Falcone, Alessandro Russo, Mario Venditti

Department of Public Health and Infectious Diseases, "Sapienza" University of Rome, Rome, Italy

Correspondence to Dr Marco Falcone, Department of Public Health and Infectious Diseases, Policlinico Umberto I, "Sapienza" University of Rome, Viale del Policlinico 155, Rome 00161, Italy; marcofalc@libero.it

Contributors MF and AR contributed to the conception of the work; MV revised it critically for important intellectual content. The final version was approved to be published. MF is responsible for the overall content.

Competing interests None.

Provenance and peer review Not commissioned; externally peer reviewed.
To cite Falcone M, Russo A, Venditti M. Thorax 2014;69:676-677.

Received 2 December 2013

Accepted 9 January 2014

Published Online First 28 January 2014

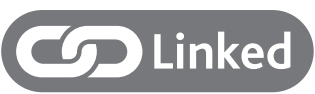

http://dx.doi.org/10.1136/thoraxjnl-2013-203828

http://dx.doi.org/10.1136/thoraxjnl-2014-205210

Thorax 2014:69:676-677.

doi:10.1136/thoraxjnl-2013-204935

\section{REFERENCES}

1 Polverino E, Torres A, Menendez R, et al.; HCAP Study investigators. Microbial aetiology of healthcare associated pneumonia in Spain: a prospective, multicentre, case-control study. Thorax 2013:68:1007-14

2 Giannella M, Pinilla B, Capdevila JA, et al. Pneumonia treated in the internal medicine department: focus on healthcare-associated pneumonia. Clin Microbiol Infect 2012;18:786-94.

3 Falcone M, Blasi F, Menichetti F, et al. Pneumonia in frail older patients: an up to date. Intern Emerg Med 2012:7:415-24.

4 Lacroix G, Prunet B, Bordes J, et al. Evaluation of early mini-bronchoalveolar lavage in the diagnosis of health care-associated pneumonia: a prospective study. Crit Care 2013;17:R24.

5 Maruyama T, Fujisawa T, Okuno M, et al. A new strategy for healthcare-associated pneumonia: a 2-year prospective multicenter cohort study using risk factors for multidrug-resistant pathogens to select initial empiric therapy. Clin Infect Dis 2013;57:1373-83. 